\title{
NOCH EINMAL GUT GEGANGEN! - DER RECHTSSTREIT ÜBER DEN DOKUMENTENLIEFERUNGSDIENST DER ETH-ZÜRICH
}

\section{von Bernhard Dengg}

Über drei Jahre hielt ein Prozess die Schweizer Bibliothekslandschaft in Atem, der zwischen der Bibliothek der ETH-Zürich und den internationalen Großverlagen Elsevier, Springer und Thieme über die Rechtmäßigkeit des Dokumentenlieferdienstes der Bibliothek geführt wurde. Als nämlich im April 2014 der erstinstanzliche Entscheid des Handelsgerichts Zürich gegen die ETH-Bibliothek ausfiel (Urteil vom 7. April 2014, HG110271-O) ${ }^{1}$, war der Aufschrei groß. Man beeilte sich von vielen Seiten, öffentlich zu der Angelegenheit Stellung zu nehmen, denn es drohte mit einer möglichen Bestätigung des Urteils in zweiter Instanz ein wesentlicher Aufgabenbereich der Bibliotheken zu Lasten der öffentlichen Informationsversorgung wegzufallen. Mit dem letztinstanzlichen Entscheid des Bundesgerichts (Urteil vom 28. November 2014, 4A-295/2014) $)^{2}$, der das Zürcher Urteil wieder aufhob und für die ETH-Bibliothek entschied, beruhigten sich die Gemüter zwar, zurück bleibt aber ein Beigeschmack: Zu klar ist die Stoßrichtung der Wissenschaftsverlage hinsichtlich der Sicherung einer ihrer Haupteinnahmequellen - nämlich der Nutzung wissenschaftlicher Aufsätze. Ob dies nur der erste oder auch schon der letzte Streich war, ist abzuwarten. Aber der Rechtsstreit zeugt jedenfalls von der Nervosität der Großverlage, die im verstärkten Aufbau von Repositorien und OpenAccess-Plattformen zunehmend eine wachsende Konkurrenz sehen.

Kernfrage des Rechtsstreits war, ob ein wissenschaftlicher Beitrag als eigenständiges Werk zu sehen ist oder nicht. Diese Frage ist entscheidend für die Auslegung der Eigengebrauchsschranke, die in Art. 19 des Schweizer Urheberrechtsgesetzes geregelt ist. Ähnlich wie $\S 42$ des österreichischen Urheberrechtsgesetzes („Vervielfältigung zum eigenen und privaten Gebrauch“) erlaubt diese Bestimmung im Sinne einer Ausnahme Vervielfältigungen im privaten sowie im betrieblichen Gebrauch sowie Vervielfältigungen zu schulischen Zwecken, wobei zu den Schulen auch die Universitäten gezählt werden. Entscheidend ist, dass eine Vervielfältigung dann nicht zulässig ist, wenn es sich um eine vollständige oder weitgehend vollständige Vervielfältigung eines im Handel erhältlichen Werkes handelt. Explizit erwähnt das Gesetz auch Bibliotheken, die als beauftragte Dritte Vervielfältigungen durchführen können. Solange man sich noch im analogen Zeitalter befand, war die Regelung trotz gelegentlicher Vorstöße von Verlagsseite oder von 
Seiten der Verwertungsgesellschaften gegen das Fotokopierwesen klar: Die zum Beispiel durch den Dokumentenlieferdienst einer Bibliothek vervielfältigten Beiträge aus Zeitschriften oder Sammelwerken wie Kongress- oder Festschriften wurden als Teile eines Gesamtwerkes gesehen.

Anders verhält es sich, seitdem die Verlage angefangen haben, ihre wissenschaftlichen Aufsätze einzeln als Download auf ihren Verlagsseiten anbieten. Obwohl dieses Angebot nun schon seit Längerem besteht, brauchte es wohl das Aufkommen von Open Access-Plattformen und Repositorien, bis die Verlage auf eine rechtliche Abklärung drängten, was als eigenständiges Werk zu gelten habe und was nicht.

Was nun das Handelsgericht Zürich feststellte, ging klar gegen das Selbstverständnis der Bibliotheken. Es bejahte nämlich die Frage der Eigenständigkeit eines Aufsatzes, sofern dieser zum Download auf Verlagsseite angeboten werde. Das öffentliche Interesse an einem Dokumentenlieferdienste wurde dabei vom Gericht verneint: Es sei nicht Kernaufgabe einer Bibliothek, einen Dokumentenlieferdienst zu betreiben, sondern ihre zentrale Aufgabe liege rein darin, ihre Bestände in ihren Räumlichkeiten zugänglich zu machen. Innerhalb der Bibliotheksräumlichkeiten, räumte das Zürcher Gericht ganz und gar nicht ironisch ein, könne durchaus mit Hilfe des Bibliothekspersonals zu Zwecken des Eigengebrauchs kopiert werden. Bei der aktuellen Handhabung des Dokumentenlieferdienstes - so das Gericht - stellten die Bibliotheken jedoch für die Rechteinhaber, also die Verlage, eindeutig eine Konkurrenz dar.

Die Konferenz der Universitätsbibliotheken der Schweiz (KUB) stellte in einer Stellungnahme ein halbes Jahr nach Veröffentlichung des Urteils mit Verweis auf die Schweizer Bibliotheksstatistik fest, dass es sich beim Dokumentenlieferdienst der ETH keinesfalls um ein Konkurrenzverhältnis handeln könnte. ${ }^{3}$ Im Verhältnis zu den ca. 39 Millionen Franken, die 2013 in der Schweiz für die Lizenzierung elektronischer Medien ausgegeben wurden, seien die 138.007 Kopieraufträge, die von den Schweizer Universitätsbibliotheken erfüllt wurden, vernachlässigbar. Hinzu komme, dass für diese Form der Vervielfältigung die Bibliotheken auch entsprechend an die Verwertungsgesellschaften finanzielle Abgaben leisteten.

In ihrer Stellungnahme wiesen die Bibliotheken auch klar auf den Schaden hin, den eine Bestätigung des Zürcher Urteils für die wissenschaftliche Literaturversorgung hätte haben können: Schlussendlich würde das Szenario darauf hinauslaufen, dass Verlage ein Vervielfältigungsmonopol bei der Digitalisierung ihrer Aufsätze zugesprochen bekommen würden.

Was dies heißen würde, war vor allem den Universitäts- und Kantonsbibliotheken von Aarau, Basel, Luzern, Solothurn und Zürich bewusst. Von 
diesen wird derzeit der Aufbau der „Kooperativen Speicherbibliothek“ in Büron, einem kleinen, abgelegenen Ort im Luzerner Entlebuch vorangetrieben. Diese Speicherbibliothek hat das Ziel, die Bestände der einzelnen Bibliotheken zusammenzuführen und auf ein Exemplar pro Titel zu reduzieren. Die Benutzung würde nur mehr über den digitalen Kopienversand erfolgen. Eine Bestätigung des Zürcher Entscheids in zweiter Instanz hätte das Projekt wohl zur Gänze zu Fall gebracht.

Ob die Stellungnahme der KUB noch Einfluss auf das Urteil des Bundesgerichts hatte, bleibe dahingestellt. Zwei Wochen nach dieser verkündete das Bundesgericht in Lausanne seinen Entscheid und hob das Zürcher Urteil wieder auf. Alles war wieder beim Alten. Das Tagesgeschäft konnte weitergehen und das zwischenzeitlich stockende Projekt der Speicherarchivierung nahm wieder Fahrt auf. Doch ein Beigeschmack bleibt.

\author{
MMag. Bernhard Dengg \\ Bereichsleiter Recht und Wirtschaft \\ Universitätsbibliothek Bern \\ E-Mail: bernhard.dengg@ub.unibe.ch
}

1 https://www.steigerlegal.ch/wp-content/uploads/2014/06/20140407 handelsgericht-zuerich urteil-hg110271-o eth-bibliothek.pdf

2 Pressemeldung: http://www.bger.ch/press-news-4a 295 2014-t.pdf; das Urteil: http://relevancy.bger.ch/php/aza/http/index. php?lang=de\&type=show document\&highlight docid=aza://28-112014-4A 295-2014

3 http://www.kub-cbu.ch/fileadmin/kub-cbu/Dokumentenlieferdienst public/Stellungnahme ETH-Prozess KUB d 20151112.pdf

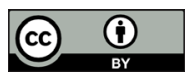

Dieses Werk ist lizenziert unter einer Creative-Commons-Lizenz Namensnennung 3.0 Österreich. 